\title{
$\mathrm{AHP}$ 를 활용한 디자인 콘셉트의 창의성 평가에 관한 연구
}

\author{
서창원 · 박영택 ${ }^{\dagger}$ \\ 성균관대학교 일반대학원 산업공학과

\section{Creativity evaluation of design concepts using AHP} \\ ChangWon Seo $\cdot$ Young T. Park ${ }^{+}$ \\ Department of Industrial Engineering, Sungkyunkwan University
}

\begin{abstract}
Purpose: Evaluation of the creativity level of design concepts.

Methods: 14 winners of design concepts area of the 2015 Red dot Award are used to evaluate the creativity level of design concepts. Among the 14 winners, one is the highest award (; luminary) winner, 4 are the second highest award(; best of the best) winners, and the remaining 9 are winners. AHP model was developed to evaluate the creativity levels of design concepts, and applied to the 14 winners of the reddot award. Results: Originality and practicality have been used to evaluate creativity level of new product ideas in the previous study. In this study, it is identified that originality is composed of innovativeness and topicality, and practicality is composed of utility and realizability. The design concepts which won higher level awards have higher level of originality. Among the two dimensions of originality, topicality has more significant relationship than innovativeness.

Conclusion: The design concepts with higher level of originality, especially higher level of topicality, have a fair chance to be recognized as creative. It is notable that higher level of originality does not guarantee higher profit. According to the previous studies on the commercial success of new products, practicality is more important than originality.
\end{abstract}

Key Words: Creativity, AHP, Design Concepts, Red Dot Design Award.

- Received 25 October 2016, 1st revised 4 December 2016, accepted 5 December 2016

+ Corresponding Author(ytpark@skku.edu)

(c) 2016, The Korean Society for Quality Management

This is an Open Access article distributed under the terms of the Creative Commons Attribution Non-Commercial License (http://creativecommons.org/licenses/by-nc/3.0) which permits unrestricted non-Commercial use, distribution, and reproduction in any medium, provided the original work is properly cited. 


\section{1. 서 론}

다양한 산업분야의 기업들은 더욱 치열해지는 경쟁에서 생존하기 위해 변화하는 고객의 니즈를 만족시켜줄 수 있 는 혁신적인 기술 및 제품개발을 핵심과제로 삼고 있다. 이에 따라 창의적인 신제품 아이디어 발상 기법에 대한 논의 가 지속적으로 이루어져 왔으며, 경영의 패러다임 또한 생산성 중시에서 창의성 경영으로 변화하고 있다.

기존 문헌 연구를 살펴보면, 아이디어 도출 부분에서 SIT(Systemic Inventive Thinking), 수렴적 사고도구, 발산 적 사고도구, TRIZ 등과 같이 다양한 아이디어 발상 기법들에 대한 연구가 많이 진행되어왔다. 그에 비해 창의적인 아이디어를 선별해 내기 위한 창의성 평가 도구 연구는 상대적으로 빈약하다.

저명한 심리학자였던 Finke(1990)는 독창성과 실용성을 지닌 제품이 창의적인 제품이라고 정의하였다. 또한 $\mathrm{Na}$ (2014)는 Finke의 연구를 토대로 창의성 평가 요인을 독창성과 실용성에 기반을 두고 있으며, 대중이 제안하는 아이디어를 제품화 시켜주는 Quirky 사의 제품 사례에 OPA(Originality-Practicality Analysis) 기법을 적용하여 아 이디어를 평가하고 선별하였다. 그러나 창의성의 평가 요인으로 사용되고 있는 독창성과 실용성이 내포하고 있는 의 미의 범위가 광범위하다는 문제점이 존재한다. 즉, 선행연구를 토대로 독창성과 실용성의 의미를 고찰하고 이를 세 분화하여 창의성 평가 척도에 대한 설명력을 높일 필요가 있다.

추가적으로 창의성 평가 시, 1점 5점까지 등간으로 설문하여 평가하는 것이 아닌 쌍대비교를 통해 설문 응답범 주의 가중치를 고려한 설문이 필요하다.

이에 따라 본 연구에서는 신제품 아이디어 선별 과정에서 창의성 평가 기준을 명확히 설정하고, AHP(Analytic Hierarchy Process) 절대측정 방식을 활용하여 각 평가요인 및 응답범주 별로 상대적인 가중치를 두어 신뢰도를 제고시킨 평가 모형을 제안한다.

레드닷 디자인상의 콘셉트 부문 수상작을 개발된 모형에 적용하고, 적용결과와 레드닷 심사 기준에 따라 평가를 거친 수상 결과를 비교·분석한다.

\section{2. 이론적 고찰}

\section{1 창의성에 관한 일반적 견해}

현대사회에서 창의성은 광범위한 영역에서 중요한 인간의 능력으로 자리매김하고 있다. 국외기업에 실시하던 창 의성 평가 면접은 최근 국내 대기업과 공공기관에서도 잇따라 도입하면서 기업 내 조직원이 갖춰야할 필수 소양으로 창의성을 내세우고 있다.

창의성에 대해 다양한 정의를 살펴보면 Amabile(1983)는 창의성은 새롭고 유용한 아이디어나 산물을 생성할 수 있는 능력이며, 어떤 아이디어나 산물이 창의적인 것으로 간주되려면 그것이 기발하고 독창적이어야 할 뿐만 아니라 유용하고 적절하며 가치가 있어야 한다고 정의하였다(Amabile 1983). Finke(1990)는 Creative Imagery라는 저서 에서 실험을 통해 창의성은 독창성(originality)과 실용성(practicality)을 가진 아이디어라고 정의하였다. Boden(1992)은 어떠한 제품이 새롭다고 판단하는 정도는 이전 제품과 얼마나 차별화되는지 그것을 받아들이는 대 중의 경험에 의해 정해진다고 하였다. 
이 후에도 창의성에 대해 다양한 정의를 내리고 있지만, 창의성에 대해 공통적인 의견은 '새롭고(독창적인) 적절한 (요구를 충족시키는) 능력'이라고 정의할 수 있다. 여기서 '새롭고'는 독창적이고 예측되지 않은 물리적 산물이나 새 로운 방식(Cropley 1999)을 의미하며, '적절한'은 유용하거나 과제의 요건을 충족시키는 것(Lubart 1994)을 의미한 다.

\section{2 창의성 평가 연구}

Finke(1990)의 창의성 실험은 현재까지 다양한 분야의 많은 연구자들에게 창의성 평가 자료로 인용되고 있다. Finke는 창의성의 구성요소를 독창성과 실용성으로 두고, 독창성과 실용성이 모두 높은 아이디어를 창의적 아이디 어라고 정의하였다. Goldenberg 등(1999; 2001)의 SIT(Systematic Inventive Thinking) 연구에서도 독창성과 실 용성을 통하여 창의성을 정량적으로 평가하였다.

$\mathrm{Kim}(2008)$ 의 디자인 창의성 연구에서 또한 창의성 평가 척도를 Finke의 독창성과 실용성 영역으로 창의성 구성 요인을 나눈 후, 그룹 회의 및 형용사 어휘 설문을 통하여 각 영역을 좀 더 세분화 하여 독창성(신규성, 흥미성), 실용 성(기능성, 현실성)으로 나누어서 디자인 분야에서 창의성 평가 척도를 구성하였다.

$\mathrm{Na}$ (2014) 또한 Finke(1990)의 독창성과 실용성을 창의성 평가 척도로 구성하고, Quirky 사에서 일반인들이 제안 한 아이디어를 상품화한 제품을 $\mathrm{OPA}$ 사분면에 타점 한 후에 창의성과 보상수익(제품 출시 후 아이디어 제공자가 받 은 수익) 간의 관계를 확인하였다. 연구 결과 창의성이 높은 제품이 보상수익이 높은 것으로 나타났으며, 독창성보다 는 실용성 점수가 높은 제품이 보상수익이 높게 나타난 것으로 확인되었다.

본 연구에서는 독창성과 실용성을 상위 기준으로 두고, 좀 더 명확히 규정하는 세부기준을 두어 $\mathrm{AHP}$ 를 활용한 상대적 중요도를 산정하여 좀 더 객관성이 제고된 창의성 평가 기준을 제안하고자 한다.

\subsection{AHP}

1970년대 초반 T. Saaty에 의하여 개발된 AHP(Analytic Hierarchy Process)는 의사결정의 계층구조를 구성하 고 있는 요소간의 쌍대비교에 의한 판단을 통하여 평가자의 지식, 경험 및 직관을 포착하고자 하는 의사결정을 지원 하는 방법론이다. AHP의 특징은 문제해결 과정에서 여러 요소의 정성적, 정량적인 측면을 모두 반영함으로써 논리 적 일관성을 갖춘 연구를 가능케 하는 것이다(Lee 2012). 또한 AHP는 문제의 구성요소를 구조화함으로써 요소들 간의 상대적인 영향력을 바탕으로 올바른 의사결정을 지원한다(Saaty 1995). 현재까지 경제, 경영, 정치, 사회, 기술 등 전 분야에 대해 AHP가 활용되고 있다(Jo 2015).

$\mathrm{AHP}$ 의 기본모형은 Figure 1 과 같다. 계층의 최상층에는 가장 포괄적인 의사결정의 목적이 놓이며, 그 다음 계층 들은 의사결정의 목적에 영향을 미치는 다양한 속성들로 구성된다. 


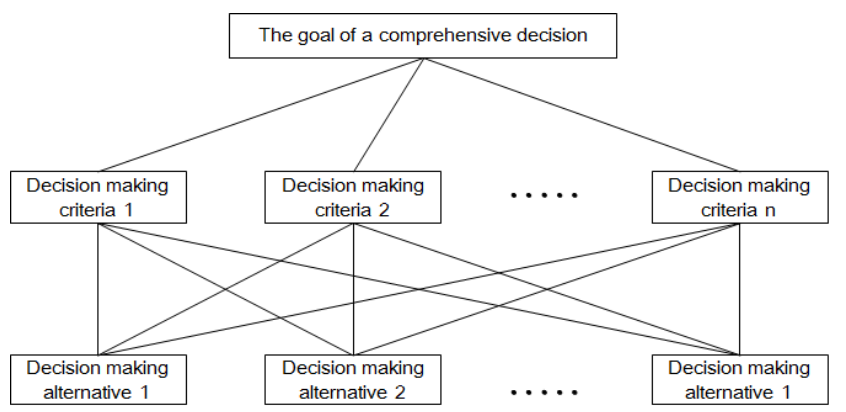

Figure 1. Basic structure of AHP

의사결정 속성은 가능하면 7개 내외로 구성한다. 의사결정의 목적에 관한 사항을 계층이 완전히 포함할 수 있도록 구성하며, 한 레벨의 요인들은 인접한 상위레벨의 요인에 대하여 종속적이어야 한다.

AHP 측정방식에는 대안의 수에 따라 상대측정, 절대측정이 있다. 대안의 수가 9 개 까지는 쌍대비교를 통해 평가 의 일관성을 어느 정도 유지할 수 있지만, 그 이상이 되면 일관성 있는 평가가 어렵다고 알려져 있다. 다양한 아이디 어의 수를 고려한 본 연구에서는 Figure 2에 따라 절대측정방식을 사용한다(Saaty 1990).

Need

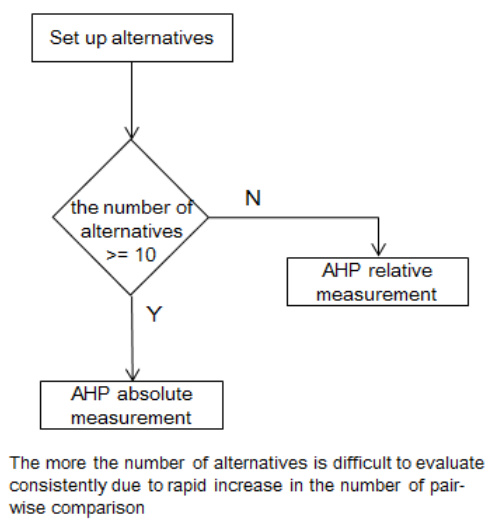

Procedure

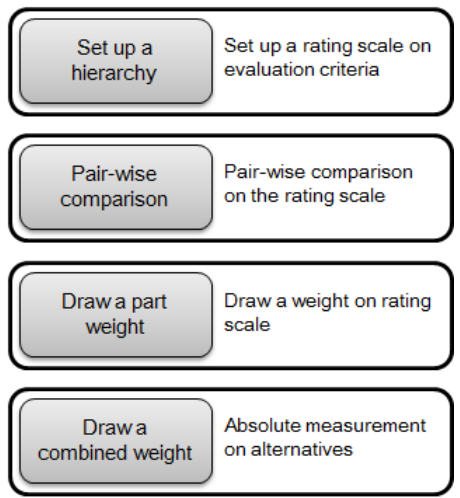

Figure 2. Absolute measurement of AHP

\section{3. 연구 모형}

\section{1 창의성 평가 기준 설정}

디자인 콘셉트에 대한 창의성 평가 기준을 설정하기 위해 본 연구는 Figure 3과 같은 프로세스를 진행하였다. 


\section{Literature reseach}
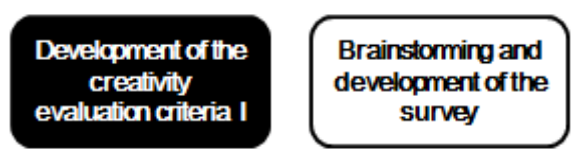

\section{Development of the creativity \\ evalualion criteriall}

Figure 3. Development process of the creativity evaluation criteria on design concepts

문헌 조사에서는 창의성 평가 기준에 대한 선행연구를 검토하고 신제품 아이디어 창의성 평가 기준에 적합한 자료 를 검색하였다. 신제품 개발 프로세스의 연구를 통해 아이디어 선별과정을 검토하고, 제품 개발 단계에서의 창의성 척도 기준 설정에 대한 근거 자료들을 수집하였다.

블루오션 전략에서 사용되는 구매자 효용성지도에서는 구매자 효용을 구성하는 차원인 효용성 지렛대를 고객 생 산성, 간편성, 편리성. 리스크, 재미와 이미지, 친환경성의 6가지 차원으로 분류하였다(Kim 2005). Jang(2009)은 구 매자 효용 지렛대를 수정하여 히트상품이 갖추고 있는 제품 속성을 도출하였고, $\mathrm{Yu}(2014)$ 는 수정된 효용성 차원 요 소를 기능성, 신뢰성, 안전성, 사용성, 심미성, 친환경성, 경제성으로 분류하였다.

디자인 측면에서 진행된 $\mathrm{Kim}(2008)$ 의 창의성 평가 연구에서는 Finke(1990)의 창의성 평가 기준인 독창성과 실 용성을 구체화 하였다. 독창성은 새로움과 흥미성, 실용성은 기능성과 현실성으로 각각 분류하여 디자인 창의성 평 가 기준을 설정하였다.

이를 토대로 해당 연구에서는 독창성과 실용성 영역에 대해 각각 대표적인 형용사 어휘를 도출하고, 도출된 어휘 에 대해 설문을 실시 한 후 요인분석으로 그룹화 하였다. 요인분석을 통해 설명력이 부족한 형용사 어휘는 제거하고, 각 형용사 어휘의 평균점수를 종합하여 혁신성, 화제성, 효용성, 실현성으로 Table 1 과 같이 최종 도출하였다.

Table 1 . The creativity evaluation criteria on design concepts

\begin{tabular}{c|c|l}
\hline Criteria & Sub-criteria & \multicolumn{1}{c}{ Keyword } \\
\hline \hline \multirow{2}{*}{ Originality } & Innovativeness & $\begin{array}{l}\text { Not existing before, applying new technology, difficult to imagine, breaking } \\
\text { a frame }\end{array}$ \\
\cline { 2 - 3 } & Topicality & Want to have, unique, attracting, something special, only one in the world \\
\hline \multirow{2}{*}{ Practicality } & Utility & Necessary, can be used anytime and anywhere, easy to use, user-friendly \\
\cline { 2 - 4 } & Realizability & Cheap, not unrealistic, safe, performing essential function, long usage time \\
\hline
\end{tabular}

각 창의성 평가 기준의 의미는 다음과 같다.

혁신성은 새로움과 기술성을 동시에 평가하며, 기존에 없던 새로움 또는 신기술 적용 측면을 고려한 특성으로 사 람들에게 새롭다는 느낌을 주는 것을 의미한다. 예를 들면 기존에 존재하지 않았거나 기존과 다르게 변형된 제품이 거나 신기술을 접목해서 나온 신상품은 “혁신성이 높다”라고 표현한다.

화제성은 희소성과 흥미유발성의 의미를 포함하며, 희소가치가 있으며 신제품이 이목을 끄는 정도를 평가한다. 예 를 들면 디자인이 예뻐서 사고 싶거나 특별한 제품인 경우 “화제성이 높다”라고 표현한다. 
효용성은 사용자의 편리함과 휴대성 측면과 제품이 기능을 잘 수행하는지에 대한 기능적 측면을 고려하여 효용성 으로 정의하였다.

실현성은 아이디어가 제품화 될 때까지 비용적 측면과 아이디어가 실제 제품화될 수 있는 지에 대한 실현가능성 측면을 포함한다. 추가적으로 안전성과 기대수명 동안 제품이 작동할 것인가에 대한 신뢰성을 포함하여 실현성으로 정의하였다.

\section{$3.2 \mathrm{AHP}$ 연구 모형 개발}

최종 도출된 창의성 평가 기준을 계층적 모형으로 Figure 4와 같이 표현하였다. 창의적 아이디어 평가를 최종 목 표로, 독창성과 실용성을 상위 기준으로, 두고 좀 더 세부적으로 독창성과 실용성을 판단하기 위해 혁신성·화제성, 효용성·실현성을 각각 하부기준에 두었다.

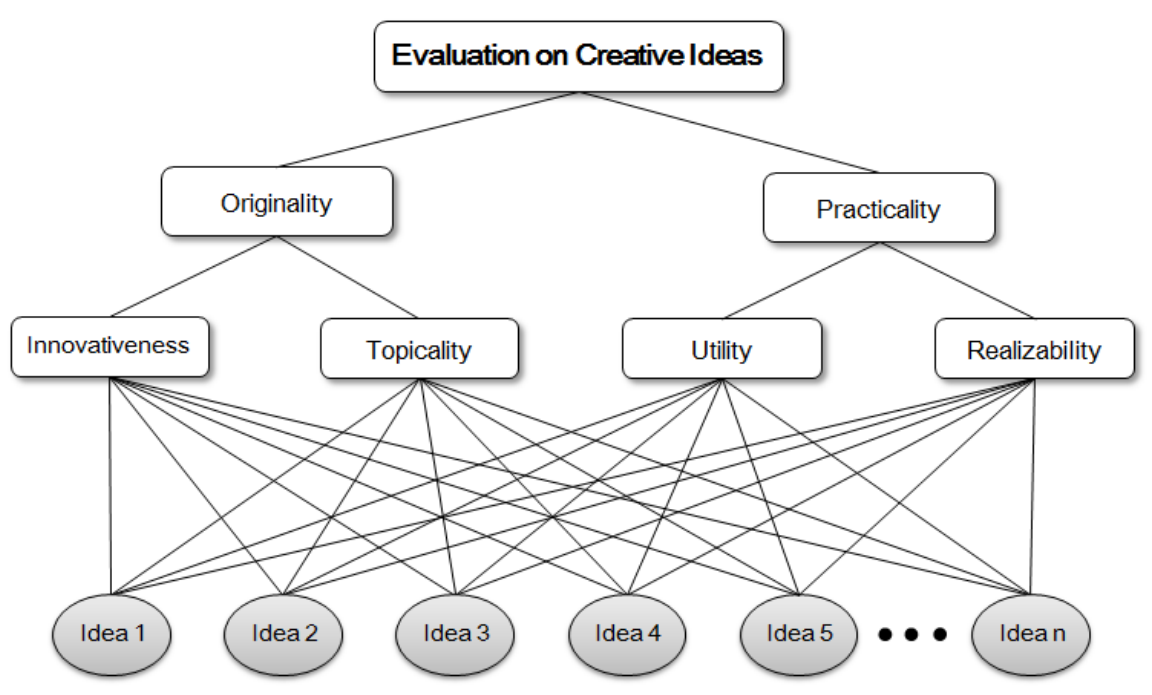

Figure 4. Hierarchy structure for evaluating creative ideas

창의성 평가 기준에 대해 합리적인 가중치를 산정하기 위해 쌍대비교 회의를 진행하였다. 쌍대비교 및 회의 진행 은 창의적 발상 과목을 이수한 대학원 학생 6 명과 함께하였다. Figure 5 는 회의 과정에서 기준에 대한 쌍대비교를 진행할 때 활용했던 설문지 샘플이다. 


\section{Comparison on evaluation criteria of creative ideas}

\begin{tabular}{|c|c|c|c|c|c|c|c|c|c|c|c|c|c|c|c|c|c|c|}
\hline & \multicolumn{8}{|c|}{ <----------------- } & & \multicolumn{8}{|c|}{$----------------->$} & \\
\hline Originality & 9 & 8 & 7 & 6 & 5 & 4 & 3 & 2 & 1 & 2 & 3 & 4 & 5 & 6 & 7 & 8 & 9 & Practicality \\
\hline
\end{tabular}

(1) Comparison on sub-criteria of originality

\begin{tabular}{|c|c|c|c|c|c|c|c|c|c|c|c|c|c|c|c|c|c|c|}
\hline & \multicolumn{1}{|c|}{ <-1 } & \multicolumn{6}{|c|}{} \\
\hline $\begin{array}{c}\text { Innoyativen } \\
\text {-ess }\end{array}$ & 9 & 8 & 7 & 6 & 5 & 4 & 3 & 2 & 1 & 2 & 3 & 4 & 5 & 6 & 7 & 8 & 9 & Topicality \\
\hline
\end{tabular}

(2) Comparison on sub-criteria of practicality

\begin{tabular}{|c|c|c|c|c|c|c|c|c|c|c|c|c|c|c|c|c|c|c|}
\hline & \multicolumn{8}{|c|}{ <------------------- } & & \multicolumn{8}{|c|}{$------------------->$} & \\
\hline Utility & 9 & 8 & 7 & 6 & 5 & 4 & 3 & 2 & 1 & 2 & 3 & 4 & 5 & 6 & 7 & 8 & 9 & Realizability \\
\hline
\end{tabular}

Figure 5. Pairwise comparison on the evaluation criteria

선행연구에서는 독창성과 실용성에 대한 상대적 중요도를 고려하지 않았으나, 본 연구에서는 각 세부 기준의 응답 범주에 대해 쌍대비교를 진행하여 상대적 가중치를 고려하였다. 단순 리커트 5점 척도 방식이 아닌 AHP 절대측정을 통해 해당 문항을 체크할 경우 가중치가 적용된 5점 척도 점수가 산정되도록 하였다.

Table 2. Pairwise comparison on the relative weights of evaluation criteria

Q. When you evaluate creativity on design concepts, please fill up the relative weights of the two corresponding levels.

\begin{tabular}{|c|c|c|c|c|c|}
\hline Innovativeness & $\begin{array}{c}\text { Very } \\
\text { Innovative }\end{array}$ & Innovative & Neutral & $\begin{array}{c}\text { Not } \\
\text { Innovative }\end{array}$ & $\begin{array}{l}\text { Very Not } \\
\text { Innovative }\end{array}$ \\
\hline $\begin{array}{c}\text { Very } \\
\text { Innovative }\end{array}$ & & 2 & 3 & 4 & 4 \\
\hline Innovative & & & 2 & 2 & 4 \\
\hline Neutral & & & & 2 & 3 \\
\hline $\begin{array}{c}\text { Not } \\
\text { Innovative }\end{array}$ & & & & & 2 \\
\hline $\begin{array}{l}\text { Very Not } \\
\text { Innovative }\end{array}$ & & & & & \\
\hline
\end{tabular}

혁신성, 화제성, 효용성, 실현성의 응답범주에 대해 각각 쌍대비교 결과를 Expert Choice를 이용하여 종합하였다. 각각의 하위기준에 대해 Table 2와 같이 쌍대비교를 진행한 후, 회의를 통하여 각 하위 기준에 대한 응답 범주의 가중치 결과를 추가한 최종 AHP모형이 Figure 6과 같이 도출되었다. 


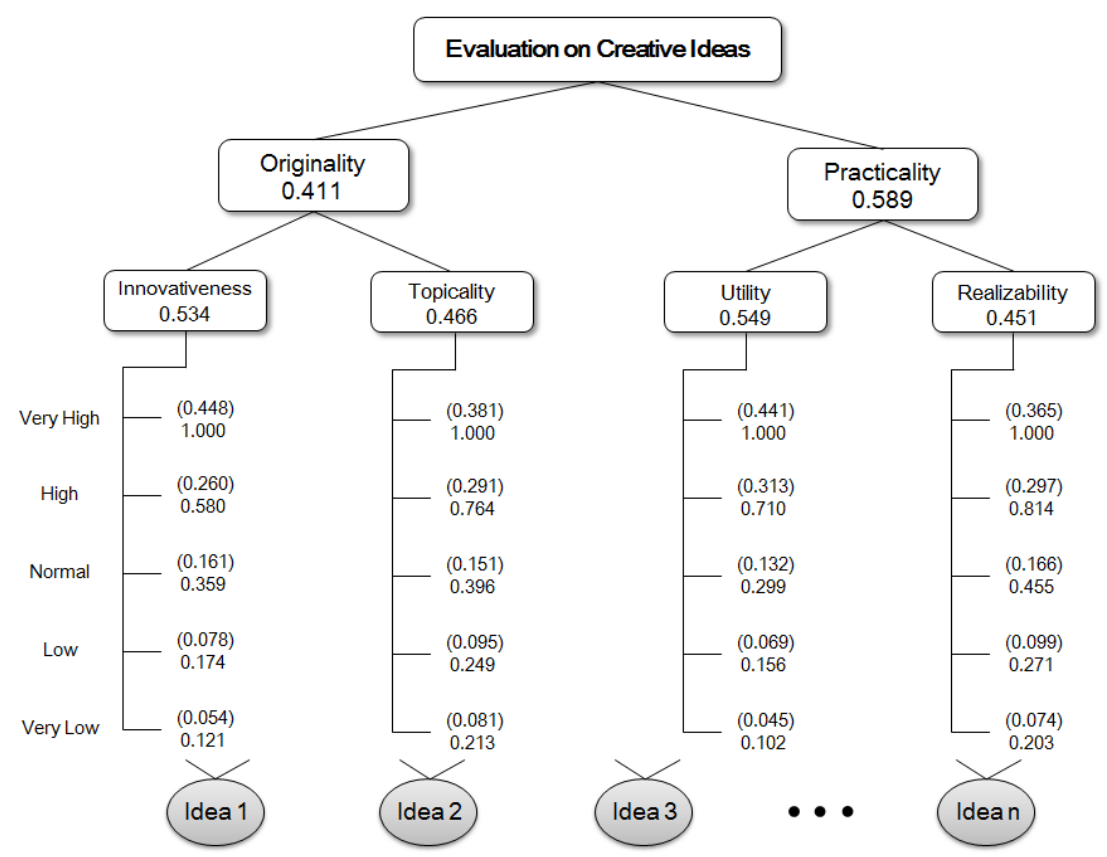

Figure 6. AHP model for evaluating creative ideas

창의성 평가 기준에 포함되는 독창성과 실용성 영역에서 살펴보면, 실용성(0.589)이 독창성(0.411)보다 좀 더 높 은 가중치로 산정되었다. 또한 독창성 영역에서는 혁신성(0.534)이 화제성(0.466)보다 좀 더 높은 가중치를 받게 되 었고, 실용성 영역에서는 효용성(0.549)이 실현성(0.451)보다 높은 가중치로 산정된 것을 확인할 수 있다.

\section{4. 모형의 적용}

\section{1 사례분석}

$\mathrm{AHP}$ 를 활용하여 새롭게 제안된 창의성 평가 모형을 적용하기 위해 레드닷 디자인 상의 디자인 콘셉트 부분의 수 상작을 연구 대상으로 한다. 레드닷 디자인 상은 기업에서 실제 출시한 제품을 평가하는 제품 부문과 제품 출시 전 아이디어를 평가하는 디자인 콘셉트 부문으로 나누어진다. 본 연구에서는 디자인 콘셉트 부문 수상작을 대상으로 모 형을 적용하였다. 레드닷 디자인 상의 디자인 콘셉트 부문의 시상 종류는 Table 3 과 같다. 
Table 3. Kinds of Red Dot Award: Design Concept Award

\begin{tabular}{l|l}
\hline Award Kinds & \multicolumn{1}{c}{ Contents } \\
\hline $\begin{array}{l}\text { The Red Dot: Luminary is the highest achievement of the Red Dot Award: Design } \\
\text { Concept. A leading light to follow, this concept is selected among the Red Dot: Best } \\
\text { of the Best winners. The Red Dot: Luminary serves as an inspiration to aspire towards } \\
\text { luminary }\end{array}$ & $\begin{array}{l}\text { The Red Dot: Best of the Best is awarded for groundbreaking design and is the top } \\
\text { prize in the Red Dot Award: Design Concept. This prize is reserved for the best works } \\
\text { in a category }\end{array}$ \\
\hline $\begin{array}{l}\text { reddot award } \\
\text { best of the best }\end{array}$ & $\begin{array}{l}\text { The Red Dot is the award for high design quality. The international jury only awards } \\
\text { this sought-after seal of quality to works that set themselves apart significantly from } \\
\text { comparable works thanks to their excellent design }\end{array}$ \\
\hline $\begin{array}{l}\text { reddot award } \\
\text { honourable mention }\end{array}$ & $\begin{array}{l}\text { An Honourable Mention recognizes particularly well-executed aspects of design work, } \\
\text { and is awarded to works with excellent detail solutions }\end{array}$ \\
\hline
\end{tabular}

분석에 활용할 사례로는 2015년도 레드닷 디자인 상의 디자인 콘셉트 부문 수상작 중 공개된 자료만으로 이해가 힘든 것을 제외한 14 개의 디자인 콘셉트 수상작을 선정하였다. 이 중 최고 등급의 Luminary 수상작은 1 개, 다음 등 급의 Best of the best은 4개, 나머지 9개는 일반 수상작이다. Honourable mention은 선정기준이 다르기 때문에 본 연구에서는 포함시키지 않았다.

$\mathrm{AHP}$ 를 활용하여 새롭게 제안된 창의성 평가 모형을 적용한 결과는 Table 4와 같다. 하위기준은 총점이 1이 되도 록 정규화 하였으며, 독창성(0.411)과 실용성(0.589)의 상대적 가중치를 적용하였다.

설문의 신뢰성을 높이기 위해 응답자들에게 디자인 콘셉트별 수상 종류를 공개하지 않았으며, 응답자 6명을 한 자리에 모은 후 디자인 콘셉트별 설명자료를 보여주면서 각각의 기준에 대해 개인적으로 응답하도록 하였다. 응답 결과는 최빈값으로 선정하였으며, 최빈값이 2 개 이상이 나올 경우 2 차 설문을 통해 합의를 도출하였다. 
Table 4. The application results of AHP

\begin{tabular}{|c|c|c|c|c|c|c|c|c|c|}
\hline \multirow[b]{2}{*}{ No } & \multicolumn{3}{|c|}{ Originality $(0.411)$} & \multicolumn{3}{|c|}{ Practicality(0.589) } & \multirow[b]{2}{*}{$\begin{array}{c}\text { Creativity } \\
\text { score }\end{array}$} & \multirow[b]{2}{*}{ Rank } & \multirow[b]{2}{*}{ Awards } \\
\hline & $\begin{array}{c}\text { Innovati } \\
\text {-veness } \\
(0.534) \\
\end{array}$ & $\begin{array}{c}\text { Topicali } \\
- \text { ty } \\
(0.466) \\
\end{array}$ & $\begin{array}{c}\text { Originali } \\
- \text { ty }\end{array}$ & $\begin{array}{l}\text { Utility } \\
(0.549)\end{array}$ & $\begin{array}{c}\text { Realizab } \\
\text {-ility } \\
(0.451) \\
\end{array}$ & $\begin{array}{c}\text { Practica } \\
\text { lity }\end{array}$ & & & \\
\hline 1 & 0.534 & 0.356 & 0.366 & 0.549 & 0.367 & 0.540 & 0.905 & 1 & Luminary \\
\hline 2 & 0.310 & 0.356 & 0.274 & 0.549 & 0.451 & 0.589 & 0.863 & 2 & $\begin{array}{c}\text { Best of the } \\
\text { best }\end{array}$ \\
\hline 3 & 0.310 & 0.356 & 0.274 & 0.549 & 0.451 & 0.589 & 0.863 & 2 & Winner \\
\hline 4 & 0.310 & 0.356 & 0.274 & 0.549 & 0.451 & 0.589 & 0.863 & 2 & Winner \\
\hline 5 & 0.534 & 0.466 & 0.411 & 0.390 & 0.367 & 0.446 & 0.857 & 5 & $\begin{array}{c}\text { Best of the } \\
\text { best }\end{array}$ \\
\hline 6 & 0.192 & 0.356 & 0.225 & 0.549 & 0.451 & 0.589 & 0.814 & 6 & Winner \\
\hline 7 & 0.310 & 0.356 & 0.274 & 0.549 & 0.367 & 0.540 & 0.813 & 7 & $\begin{array}{c}\text { Best of the } \\
\text { best }\end{array}$ \\
\hline 8 & 0.310 & 0.356 & 0.274 & 0.549 & 0.367 & 0.540 & 0.813 & 7 & Winner \\
\hline 9 & 0.534 & 0.356 & 0.366 & 0.390 & 0.367 & 0.446 & 0.811 & 9 & Winner \\
\hline 10 & 0.310 & 0.356 & 0.274 & 0.390 & 0.451 & 0.495 & 0.769 & 10 & $\begin{array}{c}\text { Best of the } \\
\text { best }\end{array}$ \\
\hline 11 & 0.310 & 0.185 & 0.203 & 0.549 & 0.367 & 0.540 & 0.743 & 11 & Winner \\
\hline 12 & 0.192 & 0.185 & 0.155 & 0.549 & 0.367 & 0.540 & 0.694 & 12 & Winner \\
\hline 13 & 0.310 & 0.356 & 0.274 & 0.390 & 0.205 & 0.350 & 0.624 & 13 & Winner \\
\hline 14 & 0.310 & 0.185 & 0.203 & 0.390 & 0.205 & 0.350 & 0.554 & 14 & Winner \\
\hline
\end{tabular}

Figure 7은 Table 4에 있는 수치들을 독창성과 실용성 영역에서 한 눈에 알아볼 수 있도록 OPA 사분면에 타점한 것이다. $\mathrm{x}$ 축은 실용성, $\mathrm{y}$ 축은 독창성이며 중간선은 각각의 기준에 대한 평균 점수를 나타낸다. 


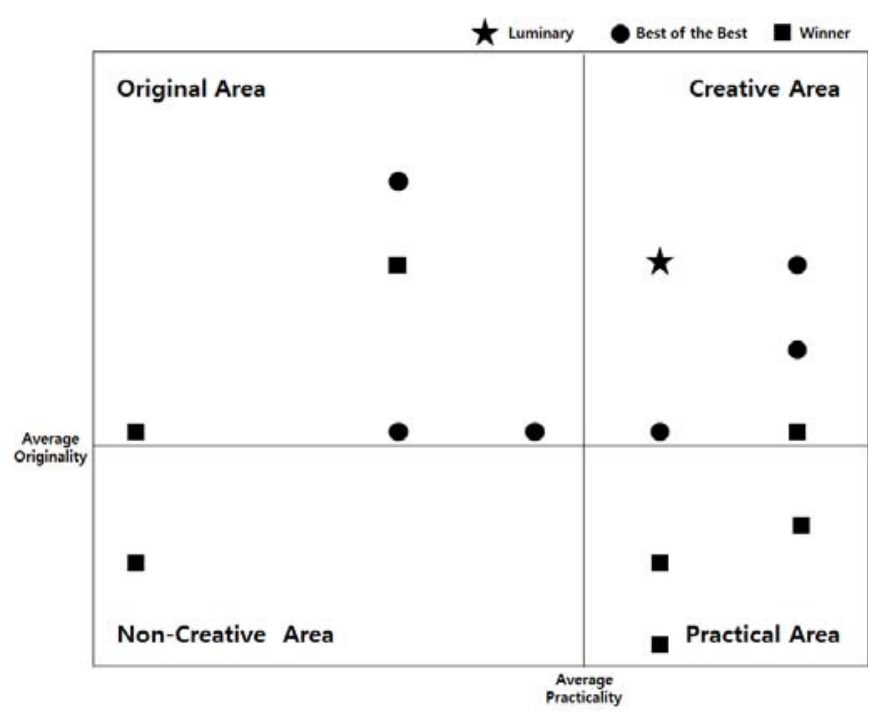

Figure 7. OPA matrix of the application results

Figure 7을 보면 높은 등급의 상인 Luminary와 Best of the best에 선정된 디자인 콘셉트들은 모두 독창성이 평균 이상인 것을 알 수 있다. 또한 최고 등급인 Luminary에 선정된 아이디어는 독창성과 실용성이 모두 평균 이상 이었다.

Table 5. The average score of originality, practicality and creativity

\begin{tabular}{c|c|c|c}
\hline 구분 & Originality & Practicality & Creativity \\
\hline \hline Luminary and Best of the best & 0.320 & 0.522 & 0.841 \\
\hline Winner & 0.250 & 0.504 & 0.753 \\
\hline
\end{tabular}

Table 5는 상위 등급의 상인 Luminary와 Best of the best에 선정된 디자인 콘셉트들과 나머지 수상작들의 독창 성과 실용성 및 이를 반영한 창의성 평가점수의 평균을 정리한 것이다. 이를 보면 상위 등급의 수상작들은 실용성보 다 독창성에서 상대적으로 높은 점수를 받은 것을 알 수 있다. Table 6은 독창성과 실용성이 상위 등급에 미치는 영향을 보기 t-검증 결과를 요약한 것이다. 
Table 6. t-test results on evaluation sub-criteria

\begin{tabular}{c|c|c|c|c}
\hline \multicolumn{2}{c|}{ Factors } & $\mathrm{t}$ & $\mathrm{Df}$ & $\mathrm{P}$-value \\
\hline \hline \multirow{3}{*}{ Originality } & Innovativeness & 1.519 & 12 & 0.155 \\
\cline { 2 - 5 } & Topicality & 1.879 & 12 & $0.049 *$ \\
\hline \multirow{3}{*}{ Practicality } & Utility & -0.231 & 12 & 0.821 \\
\cline { 2 - 5 } & Realizability & 0.904 & 12 & 0.384 \\
\hline
\end{tabular}

주 : * $\mathrm{p}<.10, * * \mathrm{p}<.05, * * * \mathrm{p}<.01$ 수준에서 통계적으로 유의함.

Table 6을 보면 독창성의 구성요소 중 혁신성보다 화제성이 상대적으로 더 큰 유의성을 갖는 것을 알 수 있다. 이것은 레드닷 디자인상의 디자인 콘셉트 부문은 제품화를 전제로 하지 않는 특성이 반영된 것으로 보인다.

\section{5. 결론 및 토의}

$\mathrm{AHP}$ 를 이용하여 디자인 콘셉트의 창의성을 평가한 본 연구를 통해 우수한 디자인 콘셉트로 평가받기 위해서는 실용성보다 독창성, 그 중에서도 화제성이 중요하다는 것을 알 수 있었다. 한 가지 주목할 점은 독창성보다 실용성이 매출수익과 주요한 요인으로 밝혀진 신제품의 창의성과 매출수익에 대한 선행연구(Heo 2015; Na 2014)와의 차이 점이다. 실용성보다 독창성이 높을 때 우수한 디자인 콘셉트로 평가받지만 이와 반대로 상업적 성과에는 독창성보다 실용성이 중요하다. 그러나 장기적인 관점에서는 독창성이 상업적 성과에 미치는 영향이 클 수 있으므로 이에 대한 심층적 연구가 필요할 것으로 생각된다.

\section{REFERENCES}

Altshuller, G. 1984. Creativity as an Exact Science. Gordon and Breach.

Amabile, T. M. 1996. "Creativity and innovation in organizations." Harvard Business Review 5.

Amabile, T. M., and Gryskiewicz, S. S. 1987. "Creativity in the R\&D Laboratory." North Carolina: Center for Creativity Leadership.

Boden, M. 1992. "The Creative Mind: Myths and Mechanisms." NewYork: Basic Books.

Clayton, M., and Scott, D. 2001. "What's the BIG Idea?" Harvard Business School Case 602-105.

Cropley, A. J. 1999. "Creativity and cognition: producting effective novelty.” Roeper Review 21:253-260.

Finke, R. A. 1990. "Creative Imagery: Discoveries and Inventions in Visualization.” NJ: Lawrence Erlbaum Associates.

Finke, R. A., and Slayton, K. 1998. "Explorations of Creative visual Synthesis in Mental Imagery." Memory \& Cognition 16(3):252-257.

Goldenberg, J., Mazursky, D., and Solomon, S. 1999. "Toward identifying the inventive templates of new products: a channeled ideation approach.” Journal of Marketing Research 36:200-210. 
Goldenberg, J., Lehmann, R.D., and Mazursky, D. 2001. "The idea itself and the circumstances of its emergence as predictors of new product success." Management Science 47:69-84.

Heo, K., Song, H. G., and Park, Y. T. 2015. "The Effect of SIT for the Development of Creative New Food: Products: Focusing on the New Products of 'H' company." Journal of the Korean Society for Quality Management 44(1):95-108.

Horowitz, R. 2001. "From TRIZ to ASIT in 4 Steps." TRIZ journal, August.

Jang, K. H. 2009. "A study on the Improvement of Buyer Utility Map in the Blue Ocean Strategy." Master thesis, Sungkyunkwan University.

Jo, K. T. 2003. The Analytic Hierarchy Process. Donghyun Publisher.

Jo, K. T., Hong, S. O., and Kwon, C. S. 2003. Decision Making for Leaders. Donghyun Publisher.

Kang, I. S., Song, H. G., and Park, Y. T. 2016. "Measuring Creativity of Ideas and the Corresponding Customer Satisfaction." Journal of the Korean Society for Quality Management 44(1):139-152.

Kim, W. C., and Renee, M. 2005. Blue Ocean Strategy. Kyobobook Publishers.

Kim, E. S. 2012. "A Comparative Study on the Effectiveness of the Creative Tools." Master thesis, Sungkyunkwan University.

Kim, E. J. 2008. "Development of the Evaluation Tool for Creativity in Design." PhD. diss., Chonbuk National University.

Lee, C. S. 2012. "An Intelligent Decision Support System for selecting Promising Technologies for R\&D based on Time-series Patent Analysis." Biblographic Info: J Intell Inform Syst 18(3):79-96.

Lubart, T. I. 1994. "Creativity Thinking and Problem Solving." New York: Academic Press.

Na, Y. S. 2015. "The Effect of New Product Creativity on Market Performance" Master thesis, Sungkyunkwan University.

Park, Y. T. 2015. "Quality Management and Creative Innovation." Journal of the Korean Society for Quality Management 43(1):1-10.

Shin, J. H. 2013. "Analysis of Buyer Utility Map in Creative Products Design." Master thesis, Sungkyunkwan University.

Stevens, G., and Burley, J. 1997. “3,000 Raw Ideas Equals 1 Commercial Sucess!” Research Technology Management 40:16-27.

Thomas L. Saaty. 1977. "A Scaling Method for Priorities in Hierarchical Structures." Journal of Mathematical Psychology 15:234-281.

Thomas L. Saaty. 1990. "How to make a decision: The Analytic Hierarchy Process." European Journal of Operational Research 48:9-26.

Yu, S. B. 2014. "A Study on SIT 5 Thinking Tools and Utility Levers." Master thesis, Sungkyunkwan University. 